\title{
Variations
}

Variations

Revue internationale de théorie critique

8 | 2006

Subjectivités libres et critique de la répression

\section{Imaginaire et désir dans l'utopie zapatiste}

Une approche de la subjectivité au temps de l'insubordination

\section{Fernando Matamoros}

Traducteur : Sylvie Bosserelle

\section{OpenEdition \\ Journals}

Édition électronique

URL : http://journals.openedition.org/variations/506

DOI : 10.4000/variations.506

ISSN : 1968-3960

Éditeur

Les amis de Variations

Édition imprimée

Date de publication : 1 novembre 2006

Pagination : 64-74

Référence électronique

Fernando Matamoros, «Imaginaire et désir dans l'utopie zapatiste», Variations [En ligne], 8| 2006, mis en ligne le 10 octobre 2012, consulté le 01 mai 2019. URL : http://journals.openedition.org/ variations/506 ; DOI : 10.4000/variations.506

Ce document a été généré automatiquement le 1 mai 2019.

Les ami•e•s de Variations 


\section{Imaginaire et désir dans l'utopie zapatiste}

Une approche de la subjectivité au temps de l'insubordination

\section{Fernando Matamoros}

Traduction : Sylvie Bosserelle

1 Le présent travail appartient à une pensée ayant surgi dans le néozapatisme et parmi de nouveaux mouvements sociaux, dans un contexte de domination des corps et des institutions. Paradoxalement, face à l'acceptation des discours homogénéisateurs du pouvoir, nous voyons apparaître dans l'histoire des vaincus une histoire de l'espoir. Ancienne et nouvelle à la fois, elle se traduit en une lutte constante et créative contre la misère des formes et contenus mercantiles du fétichisme de la marchandise et au-delà des paradigmes de la terreur néolibérale.

\section{Misère du monde, objectivation de la parole}

2 Si notre regard, formé à l'intérieur du pouvoir et de la domination (qui, par ailleurs, le constituent), veut voir au-delà des messages médiatiques de la Société du spectacle (Debord, 1992), s'il se veut extérieur, nous constaterons que, dans un contexte de guerres légitimées par les discours des vainqueurs de la guerre froide, des millions d'hommes crient aujourd'hui leur négativité. Il s'agit de l'expression de leur subjectivité faite action, contre le destin annoncé par l'ange de Walter Benjamin, luttant contre les vents du progrès (Benjamin, 2000, p. 434), nous repoussant loin de la promesse de libération et nous condamnant. L'angelus novus voudrait bien "s'attarder, réveiller les morts et rassembler ce qui a été démembré. Mais du paradis souffle une tempête qui s'est prise dans ses ailes, si violemment que l'ange ne peut plus les refermer [...] Cette tempête est ce que nous appelons le progrès. "C'est de manière chaque fois plus dramatique que les injustices sociales se révèlent dans notre monde, celui nous appartenant, et dont les autres sont exclus, dominés par le pouvoir et la fragmentation. En ce début de millénaire, la géopolitique montre que 3 personnes concentrent à elles seules le PIB de plus de 40 pays pauvres, que 
le marché est déterminé en fonction des intérêts d'environ 5000 capitalistes et hommes politiques du premier monde, directement liés à 500 entreprises transnationales, soit au total 37000 entreprises. Le résultat de cette concentration de richesses est que des 5 milliards d'habitants peuplant notre monde, seul 500 millions vivent confortablement. 358 familles ont pour revenu annuel celui de $45 \%$ des habitants des pays les plus pauvres, soit 2,6 milliards de personnes et davantage encore selon les pessimistes promesses de la vérité capitaliste (cf. Heinz Dieterich, p. 38 ; Ramonet, 1997, pp. 10-11 ; 2002, pp. 12-13). Dans sa marche visant à dominer la nature et satisfaire ses besoins, l'humanité produit aujourd'hui $110 \%$ des aliments nécessaires à satisfaire sa faim. Or, cette faim pousse des millions d'êtres humains vers l'exil, latino-américains, africains, migrants absents des discours médiatiques de la richesse, cependant à la une des peurs racistes du monde contemporain.

330 millions de personnes meurent de faim chaque année, 800 millions vivent sousalimentés. Dramatique et tragique, la surproduction oblige 1,2 milliard de personnes à survivre avec un dollar par jour, 2,8 milliards avec à peine 2 dollars. Le revenu moyen des 20 pays les plus riches de la planète est 36 fois plus élevé que celui des 20 pays les plus pauvres. Cette différence s'est démultipliée en 20 ans. Ces conséquences de l'économie néolibérale se concrétisant dans les distributions géographiques nous font orienter davantage notre critique vers le tiers-monde. Si le chômage touche 50 millions d'individus en Europe, il en touche vingt fois plus dans les pays de la périphérie, alors que 300 millions d'enfants travaillent dans des conditions intolérables. Si dans les pays riches le taux de mortalité par sous-alimentation des enfants de moins de 5 ans est de moins de $5 \%, 50 \%$ de ceux des pays pauvres meurent sans avoir atteint cet âge (Cf. Ramonet, 1999).

4 Une interprétation de ces chiffres de la misère du monde permet de saisir la subjectivité et la puissance de la parole, celles du Ya basta!, du cri contre la domination, critique et objectivation de la douleur du monde. La parole, en tant que question face au monde, est action et mouvement vers un autre monde. S'opposant à la parole du puissant, elle est " péché » face à la loi ; sécularisée, elle est aussi promesse messianique de libération. Face au mythe du progrès, son droit et ses conséquences, la douleur et la mort, la rébellion se nourrit de la parole comme libération, du divin des dieux qui l'a fait vivre et construire ces ponts sonores dont le timbre résonne en écho à celui de l'utopie. C'est elle qui est porteuse du désir et de l'espérance chez ces nouveaux pèlerins d'un pèlerinage critique du temps de la parole ici et maintenant mais dans un autre monde, "en d'autres lieux, en d'autres temps, en d'autres sociétés, vers d'autres dieux» (Desroche, 1973, p. 56). Pour reprendre Ernst Bloch (1977, p. 58), « le timbre de cette sonnerie reste dans toutes les oreilles et se mêle à chaque appel heureux du dehors [...]. Le désir lancinant de mieux tient bon et subsistera, quelles que soient les entraves qui s'imposent à ce Mieux."

C'est pourquoi des millions d'hommes et de femmes réagissent aux mythes du capital et du progrès. Dans un premier temps de rêve éveillé, brisés par la fatigue et leurs salaires misérables, nombre d'entre eux s'éloignent de l'utopie concrète du meilleur des mondes, et du rationalisme, pour imaginer un au-delà de la réalité. Bien sûr que leurs cris de révolte ne se limitent pas aux autonomies locales, à l'isolement et aux fragmentations d'identité, aux jungles paradisiaques du tourisme vert, et au nihilisme des montagnes magiques contre la mort et la condamnation. Pour ne pas mourir assassinés de solitude, d'abandon et de silence, ils affrontent leurs sentiments de déroute et d'échecs passés pour rompre avec les liens communautaires les condamnant à la fragmentation et à l'identification qui, finalement, les poussent vers l'homogénéisation du bien et du mal comme seul avenir. 
6 Pas plus qu'ils ne choisissent l'exode pour ne jamais revenir, ils ne s'intègrent à la multitude en dehors des caractéristiques sociales qui les engendrent. Ils proviennent de la fermentation du travail abstrait et de la fragmentation: la rébellion. Si l'isolement favorise qu'ils se perdent dans les réseaux des survivances culturalistes, il leur permet aussi de se situer dans les résistances locales pour s'affirmer stratégiquement et d'un point de vue utopique, en tant que sujets à l'intérieur de la globalisation capitaliste. Les réalités socio-politiques montrent qu'ils construisent leurs imaginaires et leurs discours pour se constituer au-delà du monde spécifique qui les organise. Leurs manifestations de rejet des condamnations du capital et de ses administrations, de l'État et des institutions internationales, sont des perspectives utopiques inscrites dans le conflit, le mouvement et le changement. De fait, dans l'insubordination, la subjectivité du désir devient une zone d'ombres, une région de dangers de mort faisant à la fois partie du temps s'écoulant dans ce monde et d'un autre hors de la réalité et de ses vérités. Dans la pénombre de la clandestinité, les négativités reconstruisent, à nouveau et paradoxalement, les ombres et la lumière d'un nouveau langage non répétitif. Au carrefour des chemins des certitudes de la vérité, du hasard et de l'espoir, celui de l'espérance sans vérité est un art nouveau. Tel Charon, ce passeur protecteur de l'enfer, des souffrances et de la mort, il ouvre d'autres possibles. Cependant, comme le souligne Daniel Bensaïd (1990, pp. 8-9) à propos de Walter Benjamin,

le passeur était aussi le gardien vigilant d'une ligne de partage, de démarcation.

Dans les plis nuancés et rassurants de ses capes, il dissimulait des dagues redoutables affilées. Appelant à "défricher des domaines sur lesquels la folie s'accroît jusqu'à présent », à gagner du terrain avec la hache aiguisée de la raison sans regarder ni à droite ni à gauche pour ne pas devenir la proie de l'horreur qui attire au plus profond de la forêt vierge

7 ce pacifique irréconciliable avait le goût de l'arme blanche, indispensable à toutes les résistances et les guérillas de l'esprit. Car, tout le sol devrait être défriché par la raison et être purifié des broussailles de la folie et du mythe. Pour sortir triomphant de l'angoisse de la clandestinité, car la négativité radicale se vit dans la peur, il faut sans cesse s'interroger face au risque de destruction. Les cris de mécontentement s'ébauchant à l'horizon d'un monde inconnu, à la fois proche et lointain, font partie des confrontations de classe. C'est avec l'encre de l'essence du monde, l'espoir, qu'elles dessinent les lignes de fuite du paysage conceptuel des dogmes et des lois. Dans l'essentiel de l'empirique d'en haut, les zapatistes construisent " en dessous et à gauche » des imaginaires alternatifs, présents dans les luttes et mouvements sociaux, conséquence d'une marginalisation croissante des sujets et du durcissement du système néolibéral, fait de surveillance et de répression, punition-discipline, soutenues et défendues par la guerre juste de la loi du progrès dans la sécurité et la démocratie.

\section{Violence, désir et contre-violence}

Qui sont les responsables de la violence croissante dans le monde ? L'invisible ennemi du terrorisme, les mouvements de guérilla, ces dissidents de la liberté de circulation du capital, ou bien les dogmes de la loi voulant empêcher de rêver et d'imaginer un autre monde ? Pour répondre, il est nécessaire de réfléchir sur l'abstraction que la violence fait des fins à atteindre. Une réflexion sur la distinction établie, entre fins justes et injustes dans les discours du pouvoir, implique une critique de la violence de ce même système, en tant que droit organisateur de l'injustice. Cette démarche analytique et les réponses 
possibles confirment les affirmations de Benjamin (2000, pp. 211-212) : «La violence est un produit naturel, en quelque sorte un matériau brut dont l'utilisation, sauf détournement abusif en faveur de fins injustes, ne pose aucun problème ». Les thèses benjaminiennes permettent de vérifier ce que les discours montrent au quotidien. Si tu ne te plies pas aux desseins et aux décisions de justice du capital et à ses paradigmes, démocratie et liberté - dénaturées, galvaudées, présentées comme naturelles dans les discours des médias - alors tu subiras les conséquences du mythe du droit, la guerre juste, le fouet de sa liberté. Ainsi, la justice capitaliste sera le critère et la légitimation des fins et des moyens utilisés. «Sans préjudice de cette opposition, les deux écoles se rejoignent comme le dogme fondamental commun selon lequel on peut atteindre par des moyens légitimes à des fins justes et employer des moyens légitimes pour réaliser des fins justes» (Benjamin, ibid.). Cependant, l'abstraction faite des conséquences de la violence discrétionnaire et de la misère renforce une forme de négativité. La lutte de classes poursuit son cours et montre que la domination est proportionnelle aux désirs accumulés dans l'autodétermination négative. Il n'est pas possible que la soumission, contradiction en actes qu'implique le capital, puisse s'éterniser. Chaque jour, entre feu et parole, la répression du dogme de la loi conservatrice se fragilise indirectement, face aux contre-violences, à travers un mouvement dialectique. Lorsque «la Bête», «la Grande Prostituée » institutionnelle de l'État, organisatrice et administratrice des lois du monstre capital, se trouve piégée par la croissance du désir, elle sort les griffes. Enragée, elle montre les crocs et mord, prête à tout au nom de ses privilèges, organisés par le droit et la violence. Hurlante et écumante, elle menace, attaque les brèches de l'espérance, elle blesse, elle tue ${ }^{1}$.

La dynamique issue de la contre-violence s'inscrit dans les expériences quotidiennes de la négation même du monde. Les hommes les plus ordinaires ne croient plus aux mensonges ou doutent face au silence des médias, ces institutions du mythe de la vérité. La légitimité n'est pas du côté des finalités de la domination. Le paysage de la non-croyance, ce reflet dialectique du paysage du croire ou de l'espérance, se lézarde, des fissures s'ouvrent, brèches de résistance où mûrit la subjectivité, sujet de transformation. Les résistances cessent d'être désir face à la souffrance, pour devenir stratégie autonome de l'insubordination. Elles brisent en éclats le miroir de l'homogénéité capitaliste, ouvrent des passages à la négation. John Holloway (2006, p. 19) affirme que les fissures du système peuvent être si petites que même les rebelles ne les perçoivent pas, mais lors de moments dialectiques, elles s'arment de sens, de paroles, construisent leurs chemins, demandent ou aller, inventent un nouvel art de résistance à l'intérieur et au-delà des antagonismes de la société capitaliste. Bien que configurées à l'intérieur du système, elles contiennent un " contre et au-delà » de l'ordre empirique et de sa vérité. Lors de ces mouvements, en chacun de nous existe une solidarité avec la métaphysique. Le soupir de la misère du monde parvient jusqu'au pouvoir, ses représentations, ses lois. C'est une énergie qui se potentialise dans les dynamiques de désir frustré, castré, aliéné, des programmes du spectacle de compétitivité et d'enrichissement, tuant, enterrant ou exilant l'Autre de la scène classifiée et tolérée par les institutions : les élections, les partis, les guerres, la mort. Tous sont les terrains favoris de la bête. Toujours aux aguets, elle cherche à écraser le désir, l'espoir, le changement révolutionnaire : terreur et assassinats à grande échelle ; fraude électorale organisée dans les hautes sphères techniques de l'informatique ${ }^{2}$; une autre et nouvelle guerre intergalactique et médiatique. 


\section{Les zapatistes sur scène et hors scène : l'Autre campagne}

10 Dans le monde, des millions de particules négatives gravitent dans et autour des fissures ouvertes par la résistance et l'autonomie. Le monde, ainsi que le pouvoir, réfléchit, tout comme les zapatistes avant et après le 1er janvier 1994, à de nouvelles conceptions pour changer le monde, le pays, formulant des propositions nationales et internationales (Rodríguez Lascano, 2005). Les six déclarations de la jungle lacandone sont autant un constant dialogue, renoué à travers des échos de révolte, qu'un enrichissement mutuel des solidarités, qui se rencontrent dans la constitution imaginative d'un sujet social. En effet, les expériences négatives, qui se sont renforcées et multipliées à l'extérieur grâce aux ponts jetés par les différentes subjectivités, ne se laissent plus isoler par la loi fondatrice de la violence et de la contradiction. Pour ne pas mourir seules, par le suicide ou dans l'isolement, elles recherchent des re-connaissances des différences de stratégies et d'actions. En se situant hors du monde institutionnel, avec l'Autre campagne ${ }^{3}$, les zapatistes renforcent leurs nouvelles conceptions du monde, dans une croissance des subjectivités en perspective. Ils savent que des millions de négativités éparses communiquent dans et par les brèches que le cri a ouvertes, le non à la loi, le non à l'imposition de guerres justes galactiques, le non aux bombardements propres et aseptisés que nous présentent les mass media. Toutes ces négativités s'expriment de différentes manières, toutes imaginent un autre monde, ici, maintenant, et au-delà de la réalité et de la douleur.

11 La dignité s'arme de désirs et de rêves, d'utopie : penser un autre monde. Pour ne pas amputer leur puissance imaginative, créatrice. Leur conceptualisation, basée sur les critiques sociales, inscrite dans les corps et dans les processus historiques de déroutes, d'échecs, de trahisons, ne s'affirme pas en tant que dogme ou vérité, afin d'éviter de remettre en place des principes arbitraires, à l'origine de toutes les dictatures, faits d'« absolutismes ", de conformités totalitaires (l'histoire de la honte fasciste et raciste en est pleine). Ils partent de la mémoire, d'un questionnement de l'histoire passée et présente, pour pouvoir avancer. Conditionnés par l'action et la politique du quotidien, par le poids de l'histoire, ils se construisent comme sujet de connaissance concret, mais aussi comme pensée et théorie critique radicale en prospective. Pour reprendre l'École de Francfort et, en particulier, Herbert Marcuse (Jean-Yves Martin, 2006), nous pouvons dire que la dialectique zapatiste se situe à l'intérieur du monde et de ses logiques, mais au-delà de la logique d'«assurance» de ce qui existe en tant que vérité. C'est l'insubordination pour ne plus vivre comme des "robots" de la répétition de la peur et comme des « porcs » (cf., Châtelet, 1998) mangeant les restes du capital. Ils vont au-delà du monde et de ses logiques, contredisent les sciences politiques et leurs pratiques formelles. C'est donc une critique qui révèle les modes et les contenus de la pensée classique de la stratégie, acceptée dans le passé et assimilée par le pouvoir. Ils transcendent les codes et les validations des systèmes et respirent du possible pour dépasser l'enfer de la honte de la violence.

C'est avec ironie qu'ils suivent les conseils des défunts, ne pas mourir sans espoir. Ils font voler en éclats le conformisme des totalités, admises par l'anthropologie formelle et systémique des statistiques. Leurs poèmes et légendes, supposés irréels, se moquent du système, de ses signifiants acceptés de promesses et possibilités. Ils redécouvrent des 
réalités, des significations blessées, interdites et classées attardées, sauvages ou barbares. Avec leur rébellion locale, les indigènes du Chiapas ont paradoxalement révélé que derrière le fétiche de la vérité se trouve la globalité fonctionnelle, symbolique et historique de la synthèse sociale du capital. C'est à partir du local qu'ils cherchent à se positionner sur la scène politique et sociale, pour mieux évaluer les tensions et antagonismes, latents ou déclarés, dans d'autres espaces. Différents mouvements - faisant partie de l'antagonisme de classe - surgissent ainsi d'une structure apparemment passive et docile. En faisant renaître des lieux de mémoire des signes sont imaginés, qui changent les identifications des résistances solitaires. Dans la confrontation pour construire le nous dans un espace et une territorialité expropriés par les expansions du capital, se construit aussi, imaginairement et parallèlement, un sujet avec son propre espace. C'est une question de vie ou de mort. Les Indiens ne veulent plus être l'objet de la charité, de pauvres hères dans leurs localités, leurs communautés. Ils cessent de servir aux classifications et homogénéisations de la société. Ils produisent et étendent leur espace au-delà des réserves de « bêtes exotiques » commercialisables, « humanoïdes » en voie de devenir hommes. Ils crient. Je suis Indien mais humain, je suis pauvre, mais je crée la richesse. Je suis condamné pour ce que je suis, mais j'ai l'espoir de changer le monde, cela suffit de mourir en silence dans l'isolement des montagnes, dans l'abandon des institutions. Nous voulons changer le monde pour cesser d'être nous autres, nous voulons un monde fait de tous les mondes.

13 Ainsi, les propositions du contre-pouvoir, s'affirmant dans les espaces concrets de la globalisation, ne surgissent pas hors du monde. Elles sont une accumulation de désirs de la rédemption des vaincus, elles font partie des fissures ouvertes lors du passage de l'espérance, elles sont l'héritage des traditions créatives du passé, elles sont les résistances des plus petits, des soumis au passé, mais pas pour l'éternité. Les zapatistes sont les signes négatifs des rêves hors du monde. Comme la langue et son concept Création, ils se construisent en se mettant en perspective, et à l'intérieur du concret de la catastrophe du présent. La proposition de leur parole reste inachevée, souvent incompréhensible aux logiques établies. Cependant, leur verbe, imagination de l'action concrète contre les modèles de domination, est utopie-négative dans et hors du monde. Il souligne les irrégularités du discours, ses anormalités, sa réification. Leurs clefs de la mémoire ouvrent « la boîte de Pandore ». D'un côté, ils montrent toute la misère et, de l'autre, ils libèrent l'espérance captive des conceptualisations traditionnelles, des idées reçues. Benjamin (2000, vol., I, p. 165) signale ces dimensions du divin dans la langue comme action. Leurs paroles sont le médium qui communique l'essence spirituelle des désirs, accumulés à travers les expériences. Leurs paroles sont une arme, une défense de la sentinelle, protégeant et nourrissant l'espoir contre le mythe fait loi. Les paroles exprimant la constellation présente dans notre corps, langage et mains en tant que pouvoir faire et potentialité négative est aussi signalé par Adorno (2003, p. 201) :

Percevoir la constellation dans laquelle se trouve la chose signifiée pour ainsi dire déchiffrer l'histoire que le singulier porte en lui en tant qu'advenu. De son côté, la séparation radicale de l'extérieur et de l'intérieur est conditionnée historiquement. Seul un savoir qui garde présente la valeur historique de l'objet dans son rapport aux autres objets est capable de dégager l'histoire dans l'objet; actualisation et concentration d'un déjà su qui transforme le savoir. La connaissance de l'objet dans sa constellation est celle du processus qu'il accumule en lui. Comme constellation, la pensée théorique circonscrit le concept qu'il voudrait ouvrir, espérant qu'il saute, à peu près comme les serrures des coffres-forts bien gardés: non pas 
seulement au moyen d'une seule clef ou d'un seul numéro, mais d'une combinaison de numéros.

Pour transmettre leur mécontentement, les zapatistes résistent et sortent de la clandestinité. Ils jettent des ponts arc-en-ciel de communication, des signes de feu et de parole comme subjectivité, pour réfléchir sur le monde et sa violence mythique. Ils ne veulent plus subir d'intimidation, ne plus vivre la répression policière, militaire. Ils savent qu'à tout moment le monde peut exploser, que ça suffit de vivre privé de liberté, de dignité. Pour fuir la bête sanguinaire des armes du capital, leurs subjectivités négatives continuent à rêver, en poursuivant le songe des désirs du passé, la divine promesse de rédemption. Des mots "mordants » ponctuent les conversations de Marcos et du vieil Antonio. Ils sont l'écho des "éclairs » des contes et légendes du monde indigène et méritent que l'on s'y arrête. En eux se concentre la sphère d'un autre monde, une subjectivité négative, potentialisée dans le temps, et dont les allers-retours, du passé au présent, dessinent les images du temps de résistance :

Nos ancêtres les plus âgés durent affronter l'étranger [...]. L'étranger est venu pour nous changer, nous mettre d'une autre façon, nous imposer une autre parole, une autre croyance, un autre dieu, une autre justice. L'or était son dieu, était sa croyance, sa supériorité. Le mensonge était sa parole, la cruauté sa manière d'être. Les nôtres, les plus grands guerriers les ont affrontés [...]. Des grands et bons guerriers sont tombés et morts au combat [...]. Alors, nos ancêtres les plus sages se sont réunis. Ils se racontèrent l'histoire de l'épée, de l'arbre, de la pierre et de l'eau [...]. L'épée prit la parole et dit : Je suis la plus forte et je peux vous détruire tous [... ]. Menteuse! dit l'arbre [...]. L'épée frappa et frappa jusqu'à couper le tronc et à le faire tomber. Je suis la plus forte répéta l'épée. Menteuse! dit la pierre [...]. L'épée frappa et frappa sans pouvoir la détruire mais elle la brisa en plusieurs morceaux. L'épée perdit son fil et la pierre se brisa en éclats [...]. Pendant ce temps, l'eau du ruisseau regardait la bagarre sans rien dire. L'épée la regarda et lui dit : tu es la plus faible de tous. Tu ne peux rien faire à personne [...]. Et l'épée se projeta avec force contre l'eau du ruisseau. [...] les poissons prirent peur et l'eau ne résista pas. Peu à peu, sans rien dire, l'eau reprit sa forme, recouvrit l'épée et poursuivit son chemin vers la rivière qui l'emporterait vers la grande eau que firent les dieux pour satisfaire leur soif. Le temps passa et l'épée dans l'eau commença à vieillir, à rouiller, à perdre son fil. Les poissons s'en approchaient sans peur [...]. À la fin de l'histoire, les ancêtres conclurent: nous devons quelquefois nous battre comme si nous étions l'épée face à un animal, nous devons quelquefois nous battre comme l'arbre face à la tempête, nous devons quelquefois nous battre comme les pierres face au temps. Et, en d'autres occasions, nous devons nous battre comme l'eau face à l'épée [...]. Aujourd'hui, nous devons nous transformer en eau pour suivre notre chemin vers la rivière qui nous emmène vers la grande eau où les dieux tarissent leur soif, ces dieux qui firent le monde, les premiers. C'est ainsi que firent nos ancêtres, dit le vieil Antonio. Ils résistèrent comme l'eau résista aux coups les plus féroces [...]. L'étranger, honteux, s'en fut dans un coin sans comprendre pourquoi il se sentait perdu alors qu'il avait gagné. (Marcos, 2005, pp. 80-83).

15 Ce qui est important dans leur temps, c'est d'ouvrir de nouveaux chemins, d'atteindre cette lueur ténue de l'utopie, masquée par la jungle du capital prêt à tuer ce qui bouge et avance vers un autre futur au-delà de la réalité. Sortir de l'exil, construire et vivre un autre monde que celui des névroses identitaires de la guerre quotidienne (il n'y en a pas d'autre). Charles Baudelaire (1961, pp. 303-304) parle de ce désir :

Dis-moi, mon âme, pauvre âme refroidie que penserais-tu d'habiter Lisbonne? Il doit y faire chaud, et t'y ragaillardirais comme un lézard. [...] Voilà un paysage selon ton goût; un paysage fait avec la lumière et le minéral, et le liquide pour les 
réfléchir. [...] Enfin, mon âme fait explosion, et sagement elle me crie : "N’importe où ! n'importe où ! pourvu que ce soit hors de ce monde !" accompagnés de leurs morts qu'ils rêvent d'un autre monde, et qui sont leur garantie de ne pas mourir esclaves de la condamnation car, pour reprendre Daniel Bensaïd (1990, p. 248), «Ne rien oublier nous condamnerait à mourir d'insomnie. Tout oublier nous condamnerait à la servitude sans fin de l'esclave sans mémoire ». Ils recourent à la métaphysique. Ils appellent les « fantômes » du passé, la mémoire de l'histoire dont les éclairs illuminent les chemins gris de la mort, dominante, idéologisée dans les vides de sens de la vie. Ils veulent être sujet, vivre sans mourir dans l'horreur, nier la violence heurtant le rêve et l'espérance.

C'est par les rêves que les dieux primordiaux nous parlent et nous enseignent.

L'homme qui ne sait pas rêver reste seul, il cache son ignorance dans la peur [...]. Pour qu'ils puissent parler, pour qu'ils puissent connaître et se connaître, les dieux primordiaux apprirent à rêver aux hommes et aux femmes de maïs. Ils leur donnèrent des nahuales ${ }^{4}$ pour qu'ils soient accompagnés sur le chemin de la vie. Les nahuales des vrais hommes et femmes sont le jaguar pour combattre, l'aigle pour que les rêves s'envolent, le coyote pour penser et ne pas faire cas du puissant [...]. Les dieux offrirent une lune dans chaque sein aux femmes mères pour qu'elles alimentent de rêves les nouveaux hommes et nouvelles femmes. C'est d'eux que proviennent l'histoire et la mémoire, sans eux on mange la mort et l'oubli.

La Sixième Déclaration de la Jungle Lacandone est un appel d'oxygène face au suicide de la solitude du local. C'est une nouvelle possibilité du sujet pour construire son temps et son espace d'action, pour rêver et se mobiliser au-delà de la violence du néolibéralisme. Les zapatistes ne veulent pas que l'espoir soit écrasé par le malheur d'une humanité fragmentée, piégée.

En effet, comme le souligne Charles Bukowski (1992), si l'isolement et la fragmentation détruisent, ils contribuent aussi à ce que l'on nous détruise. Il faut sortir, souffrir le monde, ses contingences; provoquer les gens et leurs traditions, changer les choses, s'organiser au quotidien pour, souvent, affronter la subjectivité des amis imbus de pouvoir, celle des ennemis jouissant des privilèges et des miettes du marché. Il faut ouvrir les portes pour que d'autres puissent entrer, là, dans la pièce où la dignité peut renaître et grandir. Malgré tout, ce n'est pas la culpabilité qui domine, c'est l'espoir qui se déploie. Comme le dit Benjamin, (1989, p. 537)

Ce n'est pas dans l'errance que l'homme se livre dans la rue; il succombe au contraire à la fascination du ruban monotone qui se déroule devant lui. Le labyrinthe représente toutefois la synthèse de ces deux types de terreur ; c'est une errance monotone.

Il nous semble que c'est pour cette raison que les zapatistes ont rejeté le recours à la violence discrétionnaire et mythique, fondatrice du droit et de sa sacralisation. Ils ont, paradoxalement, organisé une contre-violence pacifique, par l'autonomie autorisée de leur propre pouvoir. L'Armée Zapatiste de Libération Nationale est le désir et l'imagination s'organisant, d'abord comme intuition, puis comme stratégie. Elle cherche à éviter les logiques de la violence étatique car, si elle ne condamne pas la guérilla des années soixante (plusieurs de ses membres en proviennent), elle évite de tomber dans une reconversion suicidaire de guerre de guérillas qui lui sont antérieures. Les zapatistes savent, comme Benjamin (2000, p. 242), que la violence révolutionnaire est la plus pure des manifestations humaines de salut, la plus haute, ils ont pris les armes pour se faire entendre. Mais aussi, ils ont appris que leur parole fait partie de la mémoire, de l'organisation communautaire, du temps qui, plus tard, fera ce jour nouveau du nous 
constituant. Ils sont un secret divin, un bouclier, un écusson de la souveraineté, contre les exécutions sacrées du droit et de la loi constituée en État qui, par ailleurs, a récupéré les rébellions contre-hégémoniques du passé pour laver sa faute, ses crimes et mensonges, devenus vérités dans la loi et dans ses mythes constitutionnels.

\section{Temps et résistance, des rêves inachevés}

En rejetant le jeu institutionnel, l'Autre campagne de la résistance zapatiste est restée libre de tout mouvement : refus de tout financement des institutions électorales de l'État pour ne pas courir le risque de devenir une nouvelle ONG autonome locale au Chiapas. À ce prix, les zapatistes peuvent, de manière critique, générer leur temps, briser celui du capital, affronter les nouveaux mythes de la mondialisation et les utopies de la fin de l'histoire. Ils imaginent leurs propres légendes, leur cosmovision, comme des ruptures du mythe et de la chosification des relations sociales. Ils inventent de nouvelles formes de souveraineté contre le pouvoir. Poètes maudits irrévérents, ils réinventent un art, la parole, construisent des écoles et des hôpitaux (Matamoros, 2006). Tels les titans des mythes antiques, ils bousculent et renversent les limites que leur octroient le pouvoir, la nature et les dieux qu'ils affrontent. De leur temps et rêves éveillés, ils construisent la résistance, une alternative. Ils se lèvent d'entre les ruines pour donner forme au possible. Pour nourrir l'unique certitude, l'espérance, ce signe éternel; l'utopie, emblème de l'avenir.
Les anciens racontent que le Yacoñooy était un guerrier petit mais courageux et audacieux, qui n'avait peur de rien, et paraissait grand et puissant... Le soleil a ri, confiant en son pouvoir et sa force, et ignora le petit homme qui, depuis le sol, le défiait. Yacoñooy le défia de nouveau et dit ainsi: «La force de ta lumière ne m'effraie pas, j'ai pour arme le temps qui mûrit dans mon coeur ", et il raidit son arc, pointant la flèche sur le centre même du soleil arrogant. Le soleil rit de nouveau et resserra alors sa ceinture de feu autour du rebelle, pour le rendre encore plus petit. Mais le Yacoñooy se protégea avec son bouclier et résista tandis que le matin cédait la place à l'après-midi. Impuissant, le soleil voyait sa force diminuer au fil du temps, et le petit rebelle tenait bon, protégé et résistant sous son bouclier, attendant le moment propice pour l'arc et la flèche ${ }^{5}$ (Marcos, 2003).

La parole et les mots de la dignité indigène sont en devenir. Don des dieux pour le mouvement et l'action, ils font aussi partie, comme le mentionne Marcos, de la mémoire, de l'héritage des "arrière-grands-pères ». C'est la transmission d'une accumulation d'expériences contre le pouvoir du monde réel que Marcos (1997) nomme la Quatrième guerre mondiale. Le manifeste de la Sixième déclaration fait partie d'un processus de rêve inachevé de l'utopie de l'insubordination. C'est un autre "accident", un "éclair » de l'histoire. C'est un frein à la locomotive du progrès, roulant à tombeau ouvert vers l'anéantissement de l'humanité. La dialectique des images, des contes et poésies hérités du passé fait tomber le mur de la honte du livre noir de la colonisation et de l'expansion du capital (Ferro, Marc, 2003). C'est une guerre des dieux, l'espoir du pas encore utopique du carpe diem d'Ernst Bloch (vol., I, p. 367-379) qui s'expriment dans les paroles zapatistes et, au-delà, mobilisent :

Dans le monde des dieux primordiaux, ceux qui firent le monde, tout est rêve [...]. Les grands dieux vivent égaux et ensemble [...]. Le vrai monde, le grand miroir du rêve des dieux primordiaux, ceux qui créèrent le monde, est très grand et tous peuvent y vivre égaux [...]. Le monde d'aujourd'hui n'est pas juste, il n'est pas un bon miroir reflétant le monde de rêves où vivent les dieux primordiaux. C'est pour 
cela que les dieux offrirent aux hommes de maïs un miroir s'appelant dignité. Les hommes s'y voient égaux et s'ils y sont différents, ils deviennent rebelles. C'est ainsi qu'a commencé la rébellion de nos premiers grands-pères, ceux qui, aujourd'hui, meurent en nous pour que nous vivions. (Marcos, 2005). Peu importe s'ils ne sont qu'un manifeste, une façon d'affirmer notre indignation et de signer, en vue d'un changement de l'opinion dominante. La parole liée à la pensée critique n'existe pas sans l'action et sa manifestation en tant que flux social accumulé de la négation, sinon elle n'est que réification, mensonge et domaine de la vérité du statu quo et de sa loi. «Penser, c'est rester fidèle à soi tout en se niant dans de tels moments. Telle est la forme critique de la pensée. Elle seule, et non pas son entente satisfaite avec elle-même, est susceptible de l'aider à se transformer » (Adorno, 2003, p. 152).

Dans ce sens, la Sixième est une interpellation critique du temps institutionnel du pouvoir et de la domination. Elle se refuse à accepter l'empirique et le banal, «ce qui va de soi ", dans les vérités de la violence dont la structure sociale porte les stigmates. Condensation d'expériences de la pensée, ce dialogue est une nouvelle proposition - à un niveau national et international - d'unir les ponts de la pensée et de l'action politique, tous deux faisant partie des critiques de la violence institutionnelle. C'est un nouveau pari, courant le risque de perdre dans l'espoir de gagner au présent. Dans l'uniforme paysage des politiques électorales traditionnelles, des fraudes organisées et manipulées par l'empire des médias, la Sixième est un incendie vivifié par le vent d'en bas. Elle annonce des tempêtes contre le mensonge et contre le sang versé. Peut-être est-elle le prélude d'une Septième déclaration, renouvelant les espoirs et les possibilités de Révolution car, finalement, le désir, l'espoir et l'imaginaire sont plus forts que le pari sur la « chance » de la bourse, des loteries et lotos des casinos et bordels de la marchandise du capital.

\section{BIBLIOGRAPHIE}

Adorno, Theodor W., (2001) Dialectique négative, Paris, Payot.

Adorno, Theodor W., (2003), Modèles critiques, Paris, Payot.

Baudelaire, Charles, (1961), CEuvres complètes, Paris, Gallimard.

Benjamin, Walter, (1989), Paris, capitale du XIXe siècle, le livre des passages, Paris, Cerf.

Benjamin, Walter, (2000), Euvres, 3 vols, Paris, Gallimard.

Bensaïd, Daniel, (1990), Walter Benjamin, la sentinelle messianique, Paris, Plon.

Bloch, Ernst, (1976), Le Principe espérance, 3 vols., Paris, Gallimard.

Bloch, Ernst, (1977), L'Esprit de l'utopie, Paris, Gallimard.

Bukowski, Charles, (1992), Erecciones, eyaculaciones, exhibiciones, Barcelona, Anagrama.

Châtelet, G., (1998), « Relire Marcuse pour ne pas vivre comme des porcs », in Le Monde

diplomatique, août.

Variations, 8 | 2012 
Debord, Guy, (1992), La Société du spectacle, Paris, Gallimard.

Desroche, Henri, (1973), Sociologie de l'espérance, Paris, Calmann-Lévy.

Ferro, Marc, (2003), Le Livre noir du colonialisme. Paris, Hachette-Pluriel.

Heinz Dieterich, (2000), La tercera vía, México, Nuestro Tiempo.

Holloway, John, (2002), Cambiar el mundo sin tomar el poder, Buenos Aires, Herramienta-BUAP.

Holloway, John, (2006), « Un mouvement contre-et-au-delà », in Variations, printemps.

Marcos, (1997), « La quatrième guerre mondiale a commencé », in Le Monde diplomatique, www.monde-diplomatique.fr/1997/08/MARCOS/8976

Marcos, (2003), in La Jornada, México D.F., 3 février.

Marcos, (2005), Relatos del Viejo Antonio, Puebla, Espiral 7.

Martin, Jean-Yves, (2006), « Géographie et pensée d'Henri Lefèvre : pour une dialectique de la nouvelle radicalité socio-territoriale », in La Somme et le Reste, http://www.espaces-marx.org/

Matamoros Ponce, Fernando « L'Autre Campagne des zapatistes », in Le monde Diplomatique, $\mathrm{n}^{\circ}$ 623, février 2006.

Matamoros Ponce, Fernando, (1998), Mémoire et utopie au Mexique. Mythes, traditions et imaginaires dans la genèse du néozapatisme, Paris, Syllepse.

Ramonet, Ignacio, (1997), Géopolitique du chaos, Paris, Galilée.

Ramonet, Ignacio, (1999), «L'an 2000 », in Le Monde Diplomatique, Paris, décembre.

Ramonet, Ignacio, (2002), Guerres du XXIe siècle, Paris, Galilée.

Rodríguez Lascano, Sergio, « La Sexta, la razón y la ira », in Rebeldía, n³3, Mexico, juillet 2005.

\section{NOTES}

1. Voir L'Autre campagne zapatiste (Matamoros, 2006) et la violente répression des paysans d'Atenco dans l'état de Mexico, celle du corps enseignant à Oaxaca (juin 2006). Concernant Atenco, la presse a mentionné 2 morts, des douzaines de blessés, des viols et plus de 200 arrestations. Selon nos informations (http://beirut.indymedia.org/ar/2003/07/374.shtml), il y aurait eu à Oaxaca 6 morts, 4 confirmés dans un rapport de l'hôpital de l'ISSTE, parmi eux 2 enseignantes, 2 enfants, un homme adulte, un policier, des blessés graves et plusieurs arrestations.

2. De nombreux exemples en témoignent, la défaite électorale de la droite au Chili avec Salvador Allende, la mort de Mgr. Romero au Salvador ou la défaite d'Andrés Manuel López Obrador au Mexique.

3. Sexta declaración de la selva lacandona, http://www.ezln.org/documentos/2005/sexta.es.htm, juin 2005.

4. Dans les croyances méso-américaines et dans les communautés indigènes, le nahual est l'animal totem protecteur de chaque individu.

5. Cité par Matamoros Ponce (2006, p. 20). 


\section{AUTEURS}

\section{FERNANDO MATAMOROS}

Enseignant-chercheur en sociologie à l'Université de Puebla-Mexico, codirige la revue Bajo el

Volcan, auteur notamment de Mémoire et utopie au Mexique, Syllepse, 1998 